\title{
Gênese e principais precursores da Bioética
}

\author{
Genesis and main pionners of Bioethics
}

\author{
Pedro Miguel dos Santos Braga da Fonseca*
}

\begin{abstract}
Resumo
O século $X X$ ficou, indiscutivelmente, marcado por uma sucessão de acontecimentos que, para além de terem chocado o mundo, provocaram um extraordinário avanço no estudo e na análise da disciplina Bioética. Tornadas públicas as horrendas violações dos direitos fundamentais básicos do ser humano, tanto as que ocorreram durante a Segunda Guerra Mundial como as que se verificaram nos Estados Unidos (e que só foram conhecidas na década de 70), a Bioética conheceu uma destacável e notória expansão motivada pela pressão social, por impulso governamental e/ou pelo crescente interesse demonstrado por vários teóricos expertos na matéria. Esse desenvolvimento tem sido uma constante ao longo das últimas décadas. No entanto, o ponto fulcral ou de viragem no entendimento da atual Bioética deve-se à obra de Beauchamp e Childress, Principles of Biomedical Ethics, em que os autores definiram os princípios mais relevantes e de obrigatório respeito em todas as investigações e ensaios clínicos realizados sobre seres humanos.
\end{abstract}

Palavras-chave: Princípios bioéticos. Código de Nuremberg. Declaração de Helsínquia. Estudos de Tuskegee. Relatório de Belmont. Beauchamp e Childress.

\section{Abstract}

The twentieh century was marked by a succesion of events that, besides shocking the World, made possible an extraordinary advance in the study and analysis of the discipline of Bioethics. Having made public the horrendous violations of fundamental and basic rights of the human being, bot those occurred during the Second World War and those that occurred in the United States (which were only known in the 70's), Bioethics experienced an amazing and notable expansion, motivated by social pressure, government momentum and the growing interest shown by various theorists experts on the subject. This development has been a constant throughout the past decades. However, the turning point in the understanding of the actual Bioethics is due to the work of Beauchamp and Childress, Principles of Biomedical Ethics, where the authors defined the most relevant and mandatory principles in all investigations and clinical trials carried out on human subjects.

Keywords: Principles of bioethics. Nuremberg Code. Declaration of Helsinki. Tuskegee Experiment. Belmont Report. Beauchamp and Childress.

\section{Introdução}

A Bioética, entendida como "autêntica ciência multidisciplinar que tem como objetivo o estudo das consequências derivadas dos desenvolvimentos e das aplicações das Ciências Biomédicas para o ser humano em todo o seu processo vital, propondo o marco da sua licitude ética e jurídica" (ROMEO CASABONA, 1998, p. 154), tem sofrido uma enorme evolução ao longo das últimas décadas, apesar de ser uma disciplina de (relativa) recente criação. Embora pese a influência exercida por ideias e conceitos característicos de outros âmbitos e disciplinas, como podem ser os casos da ética moral, da ética social, médica ou religiosa, entendemos que o aparecimento de uma ciência que pretende estudar a posição do ser humano em relação às demais pessoas e à natureza só se verifica no século passado.

No entanto, no decorrer do século XX, a humanidade viveu certos acontecimentos que marcaram definitiva e negativamente a nossa história mais recente. Como exemplo do que acabamos de afirmar, podemos referir a Primeira Guerra Mundial (em que foram utilizadas, pela primeira vez, armas químicas em

\footnotetext{
* Doutorando em Direito pela Universidad Pablo de Olavide. Sevilla - España. E-mail: pedrobfonseca@live.com.
} 
grande escala, como o gás mostarda); a Segunda Guerra Mundial e as constantes experiências médicas realizadas sobre os prisioneiros de vários campos de concentração; e, finalmente, as experiências levadas a cabo nos Estados Unidos (Tuskegee), que apenas foram tornadas públicas em 1972. ${ }^{1}$ Foram esses nefastos episódios que conduziram a que o estudo analítico sobre a Bioética sofresse um maior impulso e acaparasse a atenção das mais variadas instituições e personalidades mundiais.

Realizada esta breve introdução, nas próximas páginas tentaremos dar ao leitor uma caracterização sucinta de alguns dos eventos que resultaram num aumento exponencial na produção teórica em torno da Bioética.

\section{As políticas de "purificação" da raça ariana durante a Segunda Guerra Mundial}

Não querendo menosprezar as consequências éticas relacionadas à utilização de armas químicas, durante o primeiro conflito, em escala planetária, não podemos negar que foi durante a Segunda Guerra Mundial que se registraram os mais aterradores abusos e violações aos direitos humanos, os quais, por sua vez, exigiram uma resposta mais firme e contundente no que diz respeito à proteção dos direitos fundamentais.

Com a chegada ao poder de Adolf Hitler, em 1933, o partido Nazi iniciou a implementação das suas políticas racistas, homófobas e xenófobas, com o objetivo de "purificar" a raça ariana, considerada pelos seus defensores como a raça superior. Judeus, indivíduos de raça cigana, doentes mentais, pessoas com deficiências físicas ou que padeciam de doenças genéticas, transmissíveis ou hereditárias, foram os que mais sofreram com a aplicação de facto da ideologia nacional-socialista. ${ }^{2}$

Dentro deste panorama que, entre 1939 e 1941, desenvolveu-se um programa de eutanásia, ${ }^{3}$ ao qual se deu o nome de Aktion T-4, que tinha como principal finalidade a aplicação prática dum conjunto de objetivos determinados e incluídos num documento assinado pelo próprio Hitler, mediante o qual se autorizava a morte de indivíduos afetados por doenças incuráveis, de crianças com perturbações hereditárias ou de adultos não produtivos ${ }^{4}$ (incluindo todos os prisioneiros dos campos de concentração que já não se encontravam em condições físicas para a execução de trabalhos forçados).

Apesar de ser oficialmente terminado em 1941, esse programa conseguiu manter-se ativo e operacional durante todo o conflito bélico. Para além das "vantagens" econômicas que esse programa Aktion T-4 apresentava, pois a eliminação de todas as pessoas doentes conduziria a uma importante diminuição do impacto financeiro que incidia sobre o sistema de saúde, permitindo canalizar esses fundos para os esforços

Se é certo que a mente humana é capaz de encontrar solução aos mais diversos problemas que afetam a sociedade - basta pensar nos avanços registrados no âmbito da Medicina -, não menos certa é a sua capacidade para cometer os atos mais cruéis e atrozes. Nesse sentido, podemos destacar a invenção da bomba atômica como exemplo dessa dicotomia. O que, inicialmente, poderia ser um incrível avanço científico, com importantes benefícios para a sociedade em geral (a descoberta da energia atômica), infelizmente, tornou-se numa das armas de maior poder destrutivo da História, poder que ficou demonstrado com os ataques a Hiroshima e Nagasaki. Especialmente reveladora dessa capacidade devastadora foi a célebre frase pronunciada por Robert Oppenheimer, principal figura do Projeto Manhattan, quando da detonação da primeira bomba nuclear (ainda em fase de testes): "Now I become Death, the destroyer of worlds".

De modo a cumprir com os planos de purificação da raça ariana, foram promulgadas várias leis cujo objetivo era a eliminação dos imperfeitos. Nesse sentido, pode mencionar-se a lei para a prevenção das doenças hereditárias da descendência que recebeu os maiores louvores por parte dos mais acérrimos defensores da eugenia. Essa lei previa a obrigação a que determinados sujeitos se submetessem a procedimentos de esterilização, especialmente em casos de pessoas que sofressem de "esquizofrenia, psicosis maníaco-depresiva, debilidad mental congénita, epilepsia hereditaria, corea de Huntington, ceguera y sordera congénita, malformaciones corporales de carácter hereditario o alcoholismo crónico". (PASCUAL; PACHO, 2015, p. 84). Os autores mencionados completam a anterior afirmação considerando que "el propósito final de éstas y otras leyes (la Ley de protección de la salud hereditaria del pueblo alemán y la Ley de salud marital, más conocidas como Leyes de Núremberg) era eliminar a una generación completa de sujetos con deficiencias genéticas a fin de "depurar" el banco de genes y mejorar la "raza aria»". (PASCUAL; $\mathrm{PACHO}, 2015$, p. 84).

3 A política e ideología nazi foram os motivos que justificaram a escolha da eutanásia, entendida como morte misericordiosa, para levar a cabo a purificação da raça ariana. Dessa forma, entende-se que "in the fall of 1939 the German government established, under the Reich Chancellery, the Euthanasie Programme under the direction of Philip Bouhler and Dr. Karl Brandt. The headquarters of the operation were at Tiergartenstrasse 4 , Berlin and the code name for the program was derived from that address - T-4. The choice of terminology for the program is consistent with the Nazis' penchant for euphemism. Euthanasia typically means 'mercy killing' and in the 1990's in the United States and other western nations, it is synonymous with 'physician-assisted suicide'. The kind of killing carried out through the T-4 program bears little resemblance to contemporary concepts of euthanasia". Disponível em: <http://www.jewishvirtuallibrary.org/background-and-overview-of-the-nazi-euthanasia-t-4-program>. Acesso em: 12 jun. 2018.

4 Conhecido como o "Decreto da Eutanásia". 
de guerra, nomeadamente para o armamento bélico, os motivos ideológicos baseados na supremacia da raça ariana levaram ao planejamento e tentativa de implementação daquela que ficaria conhecida como a "solução final", causando um dos maiores (senão o maior) genocídio da história da humanidade. ${ }^{5}$

Portanto, como acertadamente refere Romeo Casabona (1997), não é descabido afirmar que o ser humano sempre procurou o seu próprio aperfeiçoamento, tanto ao nível da sua biologia como da sua psique. ${ }^{6}$ Nesse sentido, a finalidade última da ideologia nazi era a eliminação de todos aqueles que fossem considerados "infra-humanos"', com especial incidência na raça judia. Nesse contexto, surge a figura do "doutor morte" (ou "anjo da morte"), alcunha atribuída ao Dr. Josef Mengele, ao deparar-se com um conjunto único de circunstâncias que lhe permitiam a realização de inúmeras investigações no campo da eutanásia e da eugenia. Foi no campo de concentração de Auschwitz que Mengele levou a cabo estudos e ensaios clínicos experimentais (de uma forma totalmente desumana e violadora dos princípios mais básicos em matéria de proteção dos indivíduos que se submetem a investigações médicas, assim como dos seus direitos fundamentais) centrados, principalmente, na esterilização em massa de sujeitos incapazes física e mentalmente, em homossexuais e em gêmeos (MORENO; VIVAS; DE VEGA, 2015, p. 58).

Uma vez finalizado o conflito, ditado pelo acontecido em Hiroshima e Nagasaki, as forças Aliadas passaram a julgar algumas das principais figuras do Holocausto, procedimento que ficou conhecido como os Julgamentos de Nuremberg.

\section{Do Código de Nuremberg aos princípios definidos pela Declaração de Helsínquia, de 1964}

Com o término da Segunda Guerra Mundial, as potências vencedoras ${ }^{8}$ formaram o Tribunal Militar Internacional de Nuremberg para o julgamento dos principais personagens considerados responsáveis pelas atrocidades cometidas durante o confronto armado, que resultaram na morte de milhões de pessoas. $\mathrm{Na}$ sequência dessa iniciativa, foi aprovado e publicado o estatuto do mencionado tribunal, em 8 de agosto de 1945, que foi resultado de árduas negociações entre as forças Aliadas durante a Conferência de Londres. Esse estatuto, entre outras matérias, delimitou no seu artigo $6^{\circ}$, os delitos e crimes pelos quais responderiam os nazis imputados: 1) delitos contra a paz; ${ }^{9}$ 2) crimes de guerra; ${ }^{10}$ e 3 ) crime contra a humanidade. ${ }^{11}$ Durante as audiências celebradas, foram revelados fatos e acontecimentos tão impressionantes, como os

[...] casos de experimentación en seres humanos; el programa de eutanazia nazi, experimentos com sulfonamidas, experimentos com flemones, experimentos de tifus exantemático, recolección

5 Foi durante esse período que a eugenia conheceu a sua versão mais aterradora e que permitiu diferenciar a eugenia positiva da eugenia negativa. Para Soutullo (2011, online), a eugenia é o "conjunto de métodos encaminados a mejorar la dotación genética de las poblaciones humanas o de los individuos", sendo a positiva aquela que pretende melhorar a condição do ser humano através da promoção da "propagación de los genes considerados beneficiosos" e a negativa aquela que pretende reduzir ou eliminar a transmissão de genes perjudiciais entre os seres humanos. Cabe destacar que a noção de "eugenia" teve origem na obra de Francis Galton, "Inquiries into Human Faculty" (1883) e que, segundo a opinião de Castro Moreno (2014, p. 68), pretendia caracterizar a ciência cujo objetivo primordial era melhorar a condição genética e hereditária da espécie humana.

6 Assim entende Romeo Casabona (1997, p. 106), ao considerar que "la historia de la humanidad ha conocido una permanente preocupación por la mejora del ser humano - en particular de la descendencia-, principalmente de sus características biológicas, pero también de sus potencialidades psíquicas y mentales".

Noção que incluiria pessoas de ascendência africana, ciganos, judeus, etc.

8 Estados Unidos da América, Reino Unido, França e União Soviética. No entanto, consideramos a postura assumida pelo governo dos Estados Unidos como, no mínimo, hipócrita e contraditória. Apesar do seu empenho em julgar e punir os intervenientes na execução do plano de extermínio idealizado e levado a cabo pelo partido Nazi, os serviços secretos norte-americanos iniciaram uma campanha de recrutamento de científicos alemães dentro das mais variadas áreas (química, física, médica, biologia, etc.) para o desenvolvimento de projetos relacionados com armamento nuclear, químico e biológico, e também de engenharia aeronáutica. Essa operação, sob o maior sigilo operacional, ficou conhecida como Operação Paperclip e a sua existência só seria reconhecida pela presidência americana décadas depois. Disponível em: <https://www.cia. gov/library/center-for-the-study-of-intelligence/csi-publications/csi-studies/studies/vol-58-no-3/operation-paperclip-the-secret-intelligence-programto-bring-nazi-scientists-to-america.html>. Acesso em: 24 jul. 2018.

9 Por delitos contra a paz entendem-se aqueles que implicam a "participação na planificação e realização de uma guerra de agressão violando numerosos tratados internacionais". Disponível em: <https://www.ushmm.org/ outreach/es/article.php?Moduleld=10007722>. Acesso em: 21 jul. 2018.

10 "Definidos como violações das leis e das regras internacionais acordadas para a deflagração de uma guerra". Disponível em: <https://www.ushmm. org/outreach/ptbr/article.php?Moduleld=10007722>.

11 "[...] ou seja, assassinato, extermínio, escravização, deportação e qualquer outro ato desumano cometido contra quaisquer populações civis, antes ou durante a guerra; ou a perseguição baseada em questões políticas, raciais ou religiosas, na execução de ou em conexão com qualquer crime sob a alçada deste Tribunal, estejam ou não violando as leis dos países onde sejam perpetrados". Ibídem. 
de material anatómico, experimentos psicológicos y experimentos en el área de medicina de guerra, entre otros. (ROMEO CASABONA, 1998, p. 154).

Essas condutas experimentais e atuações médico-científicas estiveram na base do julgamento de cerca de 20 pessoas (que ficou conhecido, posteriormente, como o "julgamento dos Doutores"), resultando na condenação da maioria dos imputados, e as penas impostas variaram entre as penas de prisão, entre 10 e 20 anos, de prisão perpétua e a pena de morte.

A partir desse julgamentos, foram publicados alguns documentos relacionados com os procedimentos penais, cabendo destacar o chamado Código de Nuremberg, que serviu de compêndio sobre os princípios éticos que, especialmente, a classe médica deveria respeitar em todas as situações clínicas, incluídas as experiências em seres humanos. ${ }^{12}$ Assim, influenciado diretamente pela tradição médica hipocrática e centrando-se no princípio "primum non nocere", o Código de Nuremberg formulou e formalizou um conjunto de preceitos cuja finalidade era impedir que os erros e atrocidades cometidas durante a Segunda Guerra Mundial se repetissem. Dessa forma, no âmbito da investigação médico-científica em seres humanos, considerouse imperativo o respeito e obrigatória aplicação das seguintes medidas: 1) a obtenção do consentimento informado da pessoa que se sujeitará à investigação; 2) as experiências que realizadas em seres humanos terão de ter como principal finalidade a obtenção de benefícios para o indivíduo e para a sociedade em geral; 3) deverá se experimentar, em primeiro lugar, em animais, e só quando os resultados o justifiquem, se poderá permitir a investigação e experiência em seres humanos; 4) será imperativo e indispensável que se evite ao máximo qualquer sofrimento e dano físico ou psicológico; 5) proibição de qualquer tipo de experiência em seres humanos quando a morte ou um dano irreparável sejam potenciais resultados; 6 ) ponderação obrigatória dos riscos que se assumirão e os potenciais benefícios para a sociedade; 7) adoção das medidas necessárias para a prevenção e proteção dos sujeitos que se sujeitem a uma experiência, ainda que as previsões da ocorrência de lesões incapacitantes ou morte sejam bastante reduzidas; 8 ) os procedimentos de investigação só poderão ser realizados se forem coordenados por pessoas cientificamente qualificadas; 9) se reconhecerá e respeitará o direito que assiste às pessoas de darem por terminada a experiência; 10) as pessoas cientificamente qualificadas e responsáveis pela realização da experiência deverão estar preparadas para interromper o procedimento a qualquer momento sempre que considerem que a sua continuação pode conduzir a lesões graves ou à morte do sujeito. ${ }^{13}$ Como acertadamente resume Vilchis Roa (2015, p. 93),

12 Como bem nota Almeida Neves Barbas (2007, p. 145-146), "as crueldades praticadas durante a II Guerra Mundial fizeram com que o Tribunal de Nuremberga elaborasse a primeira recomendação de condutas adequadas às investigações em seres humanos. O Código de Nuremberga foi a base de vários documentos internacionais e legislações nacionais na área da investigação. Esta discussão, de início, essencialmente, de cariz jurídico foi muito influenciada por valores morais". Em sentido similar, entende Suárez Espino $(2008$, p. 16) que este código foi "el primer documento internacional que condiciona la actividad médica al respeto de los derechos fundamentales".

13 Na sua versão em castelhano, indica o Código de Nuremberg: "Son abrumadoras las pruebas que demuestran que algunos tipos de experimentos médicos en seres humanos, cuando se mantienen dentro de límites bien definidos, satisfacen -generalmente- la ética de la profesión médica. Los protagonistas de la práctica de experimentos en humanos justifican sus puntos de vista basándose en que tales experimentos dan resultados provechosos para la sociedad, que no pueden ser procurados mediante otros métodos de estudio. Todos están de acuerdo, sin embargo, en que deben conservarse ciertos principios básicos para poder satisfacer conceptos morales, éticos y legales: 1) El consentimiento voluntario del sujeto humano es absolutamente esencial. Esto quiere decir que la persona implicada debe tener capacidad legal para dar su consentimiento; que debe estar en una situación tal que pueda ejercer su libertad de escoger, sin la intervención de cualquier elemento de fuerza, fraude, engaño, coacción o algún otro factor coercitivo o coactivo; y que debe tener el suficiente conocimiento y comprensión del asunto en sus distintos aspectos para que pueda tomar una decisión consciente. Esto último requiere que antes de aceptar una decisión afirmativa del sujeto que va a ser sometido al experimento hay que explicarle la naturaleza, duración y propósito del mismo, el método y las formas mediante las cuales se llevará a cabo, todos los inconvenientes y riesgos que pueden presentarse, y los efectos sobre su salud o persona que puedan derivarse de su participación en el experimento. El deber y la responsabilidad de determinar la calidad del consentimiento recaen en la persona que inicia, dirige, o implica a otro en el experimento. Es un deber personal y una responsabilidad que no puede ser delegada con impunidad a otra persona. 2) El experimento debe realizarse con la finalidad de obtener resultados fructíferos para el bien de la sociedad que no sean asequibles mediante otros métodos o medios de estudio, y no debe ser de naturaleza aleatoria o innecesaria.

3) El experimento debe diseñarse y basarse en los resultados obtenidos mediante la experimentación previa con animales y el pleno conocimiento de la historia natural de la enfermedad o del problema en estudio, de modo que los resultados anticipados justifiquen la realización del experimento. 4) El experimento debe ser conducido de manera tal que evite todo sufrimiento o daño innecesario físico o mental. 5) No debe realizarse experimento alguno cuando hay una razón a priori para suponer que puede ocurrir la muerte o una lesión irreparable; excepto, quizá, en los experimentos en los que los médicos investigadores son también sujetos de experimentación. 6) El riesgo tomado no debe exceder nunca el determinado por la importancia humanitaria del problema que ha de resolver el experimento. 7) Se deben tomar las precauciones adecuadas y disponer de las instalaciones óptimas para proteger al sujeto implicado de las posibilidades incluso remotas de lesión, incapacidad o muerte. 8) El experimento debe ser conducido únicamente por personas científicamente calificadas. En todas las fases del experimento se requiere la máxima precaución y capacidad técnica de los que lo dirigen o toman parte en el mismo. 9) Durante el curso del experimento el sujeto humano deber tener la libertad de poder finalizarlo si llega a un estado físico o mental en el que la continuación del experimento le parece imposible. 10) En cualquier momento durante el curso del experimento el científico que lo realiza debe estar preparado para interrumpirlo si tiene razones para creer -en el ejercicio de su buena fe, habilidad técnica y juicio cuidadoso- que la continuación del experimento puede provocar lesión incapacidad o muerte al sujeto en experimentación". Texto disponível em http://www.bioeticanet. info/documentos/ Nuremberg.pdf, consultado por última vez a13/6/18. 
El análisis del Código de Núremberg muestra que es fundamental la voluntad para ejercer la decisión de participar en un proyecto de investigación que implique experimentación científica, el voluntario debe contar con la información de los beneficios y riesgos sobre su salud, además de los objetivos, el método y los medios utilizados durante la experimentación; el sujeto de investigación tiene la libertad de retirarse de la investigación; el proyecto tiene que evitar cualquier daño, sufrimiento físico, minusvalía o muerte del participante y la importancia social no debe superar el riesgo del paciente.

Como já afirmado, os princípios consagrados no Código de Nuremberg serviram de inspiração a vários documentos posteriores. Exemplo claro do que acabamos de afirmar é a Declaração de Helsínquia, de 1964, ${ }^{14}$ realizada no âmbito da Associação Médica Mundial, que atualizou o conteúdo do Código de Nuremberg ao mesmo tempo incluindo outros novos princípios mais adequados aos avanços científicos que se iam registando. Nesse contexto, uma das inovações introduzidas no campo da investigação científica responsável e moralmente guiada pelo bem comum foi a distinção que se realizou entre a investigação terapêutica ou clínica (cuja finalidade primordial é o desenvolvimento de meios, técnicas e soluções potencialmente aplicáveis em beneficio da saúde das pessoas) e a investigação pura e estritamente científica (que visa outros objetivos que não a restrita finalidade terapêutica). Para além dessa distinção entre os dois tipos de investigação, a Declaração de Helsínquia foi relevante porque veio impor ao médico a obrigação de preservar e cuidar da saúde do paciente, independentemente de qualquer circunstância, até mesmo durante os procedimentos científicos de investigação para o desenvolvimento e aperfeiçoamento das técnicas preventivas, de diagnóstico ou terapêuticas. Dessa maneira, a investigação deve respeitar e seguir modelos cuja eficácia tenha sido anteriormente comprovada, seja com base em conhecimentos científicos adquiridos e consolidados, seja com base em resultados de experiências realizadas em laboratórios e em animais. Igualmente relevante foi a previsão sobre a formação e inclusão de Comitês de Ética de Investigação para garantir o cumprimento e execução dos princípios e obrigações éticas. A mencionada declaração determinou, também, que alguns grupos de pessoas, especialmente vulneráveis, devem ser objeto de uma concreta e específica proteção. Finalmente, haverá de mencionar a explícita imposição da obtenção do consentimento informado dos indivíduos que se submetem a uma investigação ou experiência médico-científica.

\section{A experiência norte-americana}

Apesar do horror vivido no continente europeu durante a Segunda Guerra Mundial, os Estados Unidos da América não ficaram imunes a acontecimentos que, de forma negativa, marcaram e mancharam a sua história recente. Antes dos avanços iniciais do regime Nazi, que resultariam no maior e mais sangrento conflito armado do século passado, uma pequena localidade situada no estado americano do Alabama seria testemunha involuntária de um estudo científico repugnante e de óbvia censura, que se manteve secreto durante mais de quarenta anos (só seria revelado na década de 70) e que ficaria conhecido como os Estudos de Tuskegee.

\subsection{0 estudo de Tuskegee}

Para melhor entender o contexto socioeconômico que os Estados Unidos atravessavam no início do século $X X$, haverá que se ter em conta o acentuado e vincado ambiente racista ${ }^{15}$ que, naquela época, era causa de importantes conflitos (estando a Ku Klux Klan no centro da grande maioria dos confrontos raciais), bem como a grave crise financeira motivada pelo crash da bolsa de valores de Nova York, em 1929.

14 No entanto, algum autor considera que entre ambos os documentos não existe tão estreita conexão como a que geralmente se assume. Dessa forma, argumenta De Abajo Iglesias (2001, p. 408) que "es frecuente leer que la Declaración de Helsinki tomó como base dicho Código. Pero el enfoque fue radicalmente distinto. El Código centraba su atención en los derechos del sujeto de investigación y afirmaba su consentimiento como 'absolutamente esencial', en tanto que la Declaración de Helsinki de 1964 fundaba los postulados éticos en la integridad moral y responsabilidad del médico".

15 Em que se consideravam os afroamericanos a "espécie situada mais abaixo na hierarquia Darwiniana". Num artigo publicado pelo Hastings Center, Allan M. Brandt (1978, p. 21) afirmava que, de acordo com o Dr. W. T. English, as caraterísticas físicas da população afroamericana eram indicativas dessa inferioridade. Assim, "Cranial structures, wide nasal apertures, receding chins, projecting jaws, all typed the Negro s the lowest species in the Darwinian hierarchy". 
Retomando o assunto em análise, em 1929, o Serviço de Saúde Pública dos Estados Unidos (U.S. Public Health Service, ou PHS), com o apoio financeiro do Fundo Julius Rosenwald,$^{16}$ deu início a um estudo realizado em zonas rurais do Sul do país com o fim de conhecer e determinar a incidência de sífilis na comunidade afroamericana, bem como a possibilidade de um tratamento em massa dessa mesma doença. "Comprovada" a mais alta concentração dessa doença no Condado de Macon ${ }^{17}$ (Alabama), esse lugar foi considerado o mais apropriado para testar a aplicação em massa do tratamento contra a sífilis. No entanto, com a acentuação da crise econômica de 29 , o mencionado fundo sofreu importantes limitações econômico-financeiras, originando uma acentuada redução no financiamento de diversos estudos, o que, em alguns casos, implicou sua eliminação. Este facto acabaria por afetar diretamente o Estudo de Tuskegee.

Contudo, em 1932, seguindo-se a opinião do Dr. Taliaferro Clark, ${ }^{18}$ as condições que se encontravam reunidas no Condado de Macon foram consideradas inigualáveis e insubstituíveis, já que ofereciam a oportunidade perfeita para observar os efeitos da doença. ${ }^{19}$ Como ressaltou Brandt (1978, p. 22),

From its inception, the USPHS regarded the Tuskegee Study as a classic «study in nature» rather than na experiment. As long as syphilis was so prevalent in Macon and most of the blacks went untreated throughout life, it seemed only natural to Clark that it would be valuable to observe the consequences. He described it as a 'ready-made situation'. ${ }^{20}$

Foi então que o Dr. Taliaferro Clark propôs que se levasse a cabo um ensaio clínico durante aproximadamente 8 meses. Uma vez terminado esse período e obtidas todas as informações pretendidas, os pacientes sujeitos ao dito estudo seriam, finalmente, tratados de acordo com a ars medica da época. ${ }^{21}$ Essa experiência afetaria cerca de 600 homens afroamericanos, ${ }^{22}$ com idade entre 25 e 60 anos, que seriam objeto de exames físicos para a determinação dos níveis de incidência de neuro-sífilis (se bem que não estava prevista pelo PHS a administração de tratamento aos pacientes). (BRANDT, 1978, p. 23).

No entanto, e de forma inesperada, os responsáveis pelo ensaio clínico se depararam com algumas dificuldades para encontrar sujeitos que aceitassem se submeter aos exames físicos anteriormente mencionados e, portanto, a participar da experiência que se pretendia realizar. ${ }^{23}$ Para superar esse obstáculo, decidiu-se recorrer a métodos de questionável moralidade e ética: convenceram a população de que seriam parte de um programa estatal de prevenção sanitária, ocultando-lhes a verdadeira finalidade da sua participação, o mencionado ensaio. Como explica Brandt (1978, p. 24),

They were told they were ill and were promised free care. Offered therapy, they became willing subjects. The USPHS did not tell the men that they were participants in an experiment; on the contrary, the subjects believed they were being treated for «bad blood» - the rural South's colloquialism for syphilis. The thought they were participating in a public health demonstration similar to the one that had been conducted by the Julius Rosenwald Fund in Tuskegee several years earlier.

16 "The Chicago-based Julius Rosenwald Fund was a philantropic organization dedicated to the promotion of the health, education, and welfare of Black Americans". (THOMAS; QUINN, 2000, p. 234- 235).

17 Onde se encontra a localidade de Tuskegee.

18 Na altura, Chefe da Divisão de Doenças Venéreas da PHS.

19 Em concreto, como se desenvolvia a doença em pacientes vivos, assim como o estudo dos seus cadáveres, para alcançar os resultados mais "fidedignos", suprimiu-se a aplicação dos tratamentos que, àquela altura, já eram conhecidos e aplicados na generalidade dos casos, com o intuito de deixar que a doença seguisse o seu normal desenvolvimento.

20 Além do mais, o Serviço de Saúde Pública dos Estados Unidos entendeu que o estudo poderia demonstrar que a aplicação dos tratamentos contra a sífilis não era necessária, justificando-se num estudo realizado em Oslo (Noruega). Entre 1890 e 1910 , o diretor de doenças venéreas daquela cidade do norte da Europa omitiu o tratamento a cerca de 2000 pessoas infetadas com sífilis. Assim indica Brandt (1978, p. 22-23): "It also seems that the USPHS believed the experiment might demonstrate that antisyphilitic treatment was unnecessary. As Cumming noted: «It is expected the results of this study may have a merked bearing on the treatment, or conversely the non-necessity of treatment, of cases of latent syphilis». The immediate source of Cumming's hypothesis appears to have been the famous Oslo Study of untreated syphilis. Between 1890 and 1910, Professor C. Boeck, the chief of the Oslo Venereal Clinic, withheld treatment from almost two thousand patients infected with syphilis".

21 "En EE. UU. se decidió hacer un estudio prospectivo similar al de Oslo, estudiando la incidencia de la sífilis en una zona de Alabama con alta prevalencia de sífilis, donde el 35-40\% de la población tenía serología positiva. La idea original partió del parecer de Taliaferro Clark, que propuso observar la evolución de enfermos de sífilis no tratados durante un período de 6-8 meses, pasando a continuación a tratarlos según los estándares de la época, que incluian Salvarsan y bismuto". (CUERDA-GALINDO; SIERRA-VALENTI; GONZÁLEZ-LÓPEZ; LÓPEZ-MUÑOZ, 2014, p. 850).

22 Destes 600 homens, 399 estariam infetados pela doença e os restantes, 201 não infetados, pertenciam ao que se denomina grupo de controle.

${ }^{23}$ A comunidade-alvo não acreditava que os exames físicos eram um pré-requisito para a participação no estudo, mas que se tratariam de exames de aptidão para serem recrutados e servir nas forças armadas americanas. 
Como recompensa pela sua participação, aos sujeitos se garantia atenção médica totalmente gratuita, transporte, uma refeição diária e um seguro (com um valor de \$50) para gastos em caso de morte. ${ }^{24}$

Como mencionamos acima, ainda que a utilização da penicilina como método terapêutico no combate e tratamento da sífilis fosse, naquela época, o mais comum, o dito tratamento não foi aplicado aos sujeitos, já que o estudo pretendia analisar a evolução natural e normal da doença. Quando, a 26 de julho de 1972, Jean Heller publica um artigo no jornal The New York Times ${ }^{25}$ através do qual denunciou o estudo de Tuskegee, ${ }^{26}$ informou-se que apenas 74 participantes estavam vivos e que se acreditava que possivelmente mais de 100 teriam falecido por causas especificamente relacionadas à omissão de tratamento da sífilis.

De tudo o que acabamos de referir, não será absurdo concluir que esse foi, sem dúvida, um dos momentos mais marcantes do século passado no que diz respeito à violação de direitos fundamentais do ser humano, e mais intolerável se considerará se tomamos em consideração a recente experiência vivida durante a Segunda Guerra Mundial. Como se pode explicar que se tenha consentido esse estudo durante tantos anos, depois de todos os horrores encontrados nos campos de concentração na Alemanha, Polônia, etc.?

Como oportunamente explica Brandt (1978, p. 27), "there can be little doubt that the Tuskegee researchers regarded their subjects as less than human. As a result, the ethical canons of experimenting on human subjects were completely disregarded", defendendo, ao mesmo tempo, a urgente necessidade de encontrar os meios pertinentes para a prevenção desse tipo de conduta. Explica o autor:

In retrospect the Tuskegee Study revealed more about the pathology of racism than it did about the pathology of syphilis: more about the nature of scientific inquiry than the nature of the disease process. [...] The degree of deception and damages have been seriously underestimated. As this history of the study suggests, the notion that science is a value-free discipline must be rejected. The need for greater vigilance in assessing the specific ways in ehich social values and attitudes affect profesional behavior is clearly indicated (BRANDT, 1978, p. 27).

\subsection{A National Comission for the Protection of Human Subjects of Biomedical and Behavioral Research e o Relatório de Belmont}

Como consequência da publicação do artigo de Heller denunciando o ocorrido durante cerca de 40 $\operatorname{anos}^{27}$ (e, certamente, influenciado pelo contexto sócio-político que se vivia na sociedade americana), ${ }^{28} \mathrm{O}$

24 A falta de moralidade e ética do programa, bem como a sua frieza, ficou bem patente no interesse que os responsáveis pelo estudo demonstraram pelo "valor" científico atribuido aos cadáveres. Chegaram a afirmar que os cadáveres dos participantes tinham mais importância que os participantes vivos. A essa conclusão chegamos a partir das palavras de Brandt, ao revelar que, numa das cartas enviadas por um dos responsáveis pela experiência, se considerava que os pacientes não teriam qualquer tipo de interesse, pelo menos até a sua morte. Indica Brandt (1978, p. 24): “As I see it' responded Wenger, "we have no further interest in these patients until they die». Apparently, the physicians engaged in the experiment believed that only autopsies could scientifically confirm the findings of the study".

25 A notícia, "Syphilis Victims in U.S. Study Went Untreated for 40 Years", pode ser consultada em: <http://www.nytimes.com/1972/07/26/archives/ syphilis-victims-in-us-study-went-untreated-for-40-years-syphilis.html>.

26 Jean Heller obteve a informação relativa à existência do estudo de Peter Buxtun que, anos antes, tinha tentado terminar a experiência que se realizava em Tuskegee. No entanto, a sua posição e pretensão foram denegadas, o que o levou a denunciar publicamente o que se estava passando na pequena localidade do Condado de Macon, esperando que, com a repulsa e pressão social, os responsáveis pelo estudo finalizassem definitivamente essas experiências reprováveis.

27 Infelizmente, o Estudo de Tuskegee não foi o único caso que assombrou os Estados Unidos, existindo outros acontecimentos que também provocaram um tremendo escândalo e horror: o caso da Escola Estatal de Willowbrook, em que crianças com deficiências mentais foram expostas, deliberada e intencionalmente, a hepatite (estudo conduzido entre 1963 e 1966); o ensaio clínico no Hospital Judeu de Doenças Crônicas de Brooklyn, que implicou a injeção de células cancerígenas vivas em pacientes idosos sem o seu consentimento; o caso Milgram, que pretendia estudar a obediência, e que foi considerado extremamento reprovável por causa dos potenciais e mais que prováveis danos psicológicos provocados (os indivíduos davam choques eléctricos a outros sujeitos quando esses últimos se enganavam nas respostas às perguntas que lhes eram colocadas); e o também repugnante caso das experiências sobre os efeitos da radiação nos seres humanos, que consistia em detonar bombas nucleares de forma a analisar os efeitos dos elementos radioativos (especialmente o iodo-131) nas populações afetadas (esses elementos radioativos eram os principais causantes de vários tipos de cancro). (VELASCO GOGENOLA, 2009, p. 236). Finalmente, não nos podemos esquecer as experiências levadas a cabo pela Agência Central de Inteligência (a CIA) em que eram utilizadas determinadas drogas (como a LSD) para obter um total control sobre a vontade e a mente dos sujeitos. Esses procedimentos, que na sua grande maioria careciam de consentimento das pessoas afetadas, ficou conhecida como Projeto MKUltra.

28 Como motivos dessa instabilidade podemos indicar o escândalo de Watergate (a entrada ilegal na sede do Commité Nacional Demócrata por pessoas relacionadas com o Presidente Nixon e que resultou no seu impeachment) e a Guerra do Vietnam, alvo generalizado de crítica e censura social e política. 
então Presidente Richard Nixon assinou e promulgou a National Research Act, em 12 de julho de 1974, ${ }^{29}$ que previa a formação da Comissão Nacional para a Proteção dos Sujeitos Humanos na Investigação Biomédica e Comportamental. ${ }^{30} \mathrm{~A}$ principal finalidade dessa comissão era a identificação, formulação e consagração de um conjunto básico de princípios éticos que seriam de imperativo respeito e observância em ensaios clínicos e investigações biomédicas e comportamentais que envolvessem a participação de seres humanos. Para tal, incumbia à mencionada comissão estabelecer de maneira clara: 1) as fronteiras entre as investigações biomédicas e comportamentais, bem como as práticas médicas permitidas; 2) quais os critérios de risco e benefício mais apropriados no âmbito das investigações em seres humanos; 3) as orientações a seguir nos processos de seleção dos sujeitos humanos para a sua participação e colaboração na investigação; 4) a definição e natureza do consentimento informado ao longo do processo de investigação. ${ }^{31}$

Assim, é dentro desse enquadramento jurídico e ético-científico que se prepara o Relatório de Belmont sobre os princípios éticos e pautas para a proteção dos seres humanos na investigação, ${ }^{32}$ o qual adotou o nome do Centro de Conferências onde o documento foi discutido. Esse relatório acabaria por ser publicado em 1979, e ainda hoje é considerado um dos principais documentos no que diz respeito à previsão dos princípios éticos e bioéticos mínimos e indispensáveis no âmbito da investigação e experimentação realizada em seres humanos. O Relatório de Belmont justificou a sua elaboração na insuficiência e ineficácia dos códigos éticos existentes na época, na constante e recorrente violação dos direitos fundamentais, e na dificuldade na interpretação e aplicação prática das suas normas. ${ }^{33}$ Portanto, para eliminar definitivamente esses obstáculos, foram consagrados três princípios, de caráter injuntivo, cuja aplicação se impõe ao longo de todas as etapas e fases dos processos investigativos.

O primeiro princípio que se declarou foi o de respeito pelas pessoas, o qual exige, por um lado, a consideração dos seres humanos participantes na investigação como seres autônomos ${ }^{34} \mathrm{e}$, por outro lado, exige uma especial proteção e tutela daqueles indivíduos com menor capacidade ou que, por motivos

29 Cujo título completo é "An Act to amend the Public Health Service Act to establish a program of National Research Service Awards to assure the continued excellence of biomedical and behavioral research and to provide for the protection of human subjects involved in biomedical and behavioral research and for other purposes".

30 Esta Comissão elaborou sendos Relatórios no âmbito da responsabilidade ética e bioética em investigação biomédica: "Research on the Fetus (1975), Research Involving Prisoners (1976), Research Involving Children (1977), Psychosurgery Report and Recommendations (1977), Disclosure of Research Information Under the Freedom of Information Act (1977), Research Involving Those Institutionalized as Mentally Infirm (1978), Ethical Guidelines for the Delivery of Health Services by DHEW (1978), Appendix to Ethical Guidelines for the Delivery of Health Services by DHEW (1978), Institutional Review Boards (1978), Special Study Implications of Advances in Biomedical and Behavioral Research (1978) y The Belmont Report: Ethical Principles and Guidelines for Protection of Human Subjects of Biomedical and Behavioral Research (1979)". (ÁLVAREZ-DíAZ, 2015, p. 154).

31 "One of the charges to the Commission was to identify the basic ethical principles that should underlie the conduct of biomedical and behavioral research involving human subjects and to develop guidelines which should be followed to assure that such research is conducted in accordance with those principles. In carrying out the above, the Commission was directed to consider: (i) the boundaries between biomedical and behavioral research and the accepted and routine practice of medicine, (ii) the role of assessment of risk-benefit criteria in the determination of the appropriateness of research involving human subjects, (iii) appropriate guidelines for the selection of human subjects for participation in such research, and (iv) the nature and definition of informed consent in various research settings". O texto completo do Relatório de Belmont, na sua versão original, pode ser consultado em: https://www.hhs.gov/ohrp/regulations-and-policy/belmont-report/read-the-belmont-report/index.html>. Acesso em: 13 maio 2018.

32 Na versão original, o título completo do Relatório é "Belmont Report: Ethical Principles and Guidelines for the Protection of Human Subjects of Research, Report of the National Comission for the Protection of Human Subjects of Biomedical and Behavioral Research".

33 Já dizia o Relatório: "Scientific research has produced substantial social benefits. If has also posed some troubling ethical questions. Public attention was drawn to these questions by reported abuses of human subjects in biomedical experiments, especially during the Second World War. During the Nuremberg War Crime Trials, the Nuremberg Code was drafted as a set of standards for judging physicians and scientists who had conducted biomedical experiments on concentration camp prisoners. This code became the prototype of many later codes intended to assure that research involving human subjects would carried out in an ethical manner. The codes consist of rules, some general, others specific, that guide the investigators or the reviewers of research in their work. Such rules often are inadequate to cover complex situations: at times they come into conflict, and they are frequently difficult to interpret or apply. Broader ethical principles will provide a basis on which specific rules may be formulated, criticized and interpreted".

34 Autonomia aqui entendida como a capacidade decisória dos indivíduos em relação a todos os aspectos da sua vida ou relacionados com a sua própria pessoa. 
concretos, tenham a sua autonomia reduzida ou limitada. ${ }^{35} \mathrm{O}$ segundo princípio estipula que toda a atuação médica deve estar orientada ao tratamento ético dos pacientes, protegendo-os de qualquer dano que possa ser previsível, e à garantia do seu bem-estar: ao que se denominou princípio da beneficiência. ${ }^{36}$ Finalmente, o princípio de justiça, que Guillem-Tatay (2013, online) caracteriza e resume da seguinte forma:

JUSTICIA: Para explicar este principio, el Informe comienza por una serie de preguntas: ¿quién debe ser el beneficiario de la investigación y quién debería sufrir sus cargas? Este es un problema de justicia, entendida como 'equidad en la distribución' o 'lo que es merecido'. Se debe impedir, en todo caso, que la investigación sólo sirva para aquellos que pueden pagar el medicamento en cuestión. Por otro lado, existen varias formulaciones ampliamente aceptadas sobre la justa distribución de cargas y beneficios:

\section{A cada persona una parte igual.}

A cada persona según su necesidad individual.

A cada persona según su propio esfuerzo.

A cada persona según su contribución a la sociedad.

A cada persona según su mérito. ${ }^{37}$

Como última apreciação no que concerne ao Relatório de Belmont, temos de destacar a importância que se atribuiu ao consentimento informado como expressão do princípio de respeito pelas pessoas que se submetem a qualquer investigação, experiência ou ensaio clínico. Nesse sentido, e como forma de garantir a possibilidade de decidir acerca das atuações médicas que poderão influir no seu próprio bem-estar, ${ }^{38}$ considera-se inegável o direito que assiste aos participantes de consentir, ato esse que será caracterizado pela necessária concorrência de três requisitos indispensáveis para a sua validez: em primeiro lugar, terá

5 "Respect for Persons. - Respect for persons incorporates at least two ethical convictions: first, that individuals should be treated as autonomous agents, and second, that persons with diminished autonomy are entitled to protection. The principle of respect for persons thus divides into two separate moral requirements: the requirement to acknowledge autonomy and the requirement to protect those with diminished autonomy". Na opinião de Guillem-Tatay (2013, online), o princípio de respeito carateriza-se por incluir "dos concreciones éticas: que todos los individuos sean tratados como agentes autónomos, es decir, capaces de deliberar sobre sus fines personales y obrar bajo la dirección de estos, por lo que hay que respetar esa autonomía y no poner obstáculos a la misma, salvo que los criterios por los que se rija sean perjudiciales para los demás: la otra concreción ética es que todas las personas cuya autonomía esté disminuida, tienen derecho a protección. Por último, este principio exige, en el caso de investigación con seres humanos, voluntariedad e información".

36 O princípio de beneficiência é definido por Guillem-Tatay $(2013$, online) como "aquellos actos de bondad y caridad que van más allá de la obligación estricta'. Este principio tiene dos reglas complementarias: por un lado, no causar ningún daño y, por otro lado, maximizar los beneficios y disminuir los posibles daños". Para o Relatório de Belmont, esse princípio implica que "Persons are treated in an ethical manner not only by respecting their decisions and protecting them from harm, but also by making efforts to secure their well-being. Such treatment falls under the principle of beneficence. The term 'beneficence' is often understood to cover acts of kindness or charity that go beyond strict obligation. In this document, beneficence is understood in a stronger sense, as an obligation. Two general rules have been formulated as complementary expressions of beneficent actions in this sense: (1) do not harm and (2) maximize possible benefits and minimize possible harms". De forma bastante resumida, Almeida Neves Barbas (2007, p. 139) define o princípio da beneficiência como aquele que se apoia numa avaliação e ponderação dos riscos e dos benefícios. Por nossa parte, entendemos que esse príncipio se associa e relaciona intimamente com a ideia latina de primum non nocere, e que tem estado presente ao longo da história médica, especialmente a partir do Juramento de Hipócrates.

37 O Relatório de Belmont, por outra parte, indica, textual e integralmente: "Justice. -Who ought to receive the benefits of research and bear its burdens? This is a question of justice, in the sense of 'fairness in distribution' or 'what is deserved.' An injustice occurs when some benefit to which a person is entitled is denied without good reason or when some burden is imposed unduly. Another way of conceiving the principle of justice is that equals ought to be treated equally. However, this statement requires explication. Who is equal and who is unequal? What considerations justify departure from equal distribution? Almost all commentators allow that distinctions based on experience, age, deprivation, competence, merit and position do sometimes constitute criteria justifying differential treatment for certain purposes. It is necessary, then, to explain in what respects people should be treated equally. There are several widely accepted formulations of just ways to distribute burdens and benefits. Each formulation mentions some relevant property on the basis of which burdens and benefits should be distributed. These formulations are (1) to each person an equal share. (2) to each person according to individual need, (3) to each person according to individual effort, (4) to each person according to societal contribution, and (5) to each person according to merit. Questions of justice have long been associated with social practices such as punishment, taxation and political representation. Until recently these questions have not generally been associated with scientific research. However, they are foreshadowed even in the earliest reflections on the ethics of research involving human subjects. For example, during the 19th and early 20th centuries the burdens of serving as research subjects fell largely upon poor ward patients, while the benefits of improved medical care flowed primarily to private patients. Subsequently, the exploitation of unwilling prisoners as research subjects in Nazi concentration camps was condemned as a particularly flagrant injustice. In this country, in the 1940's, the Tuskegee syphilis study used disadvantaged, rural black men to study the untreated course of a disease that is by no means confined to that population. These subjects were deprived of demonstrably effective treatment in order not to interrupt the project, long after such treatment became generally available. Against this historical background, it can be seen how conceptions of justice are relevant to research involving human subjects. For example, the selection of research subjects needs to be scrutinized in order to determine whether some classes (e.g., welfare patients, particular racial and ethnic minorities, or persons confined to institutions) are being systematically selected simply because of their easy availability, their compromised position, or their manipulability, rather than for reasons directly related to the problem being studied. Finally, whenever research supported by public funds leads to the development of therapeutic devices and procedures, justice demands both that these not provide advantages only to those who can afford them and that such research should not unduly involve persons from groups unlikely to be among the beneficiaries of subsequent applications of the research".

38 Diz o Relatório: "1. Informed Consent. -Respect for persons requires that subjects, to the degree that they are capable, be given the opportunity to choose what shall or shall not happen to them. This opportunity is provided when adequate standards for informed consent are satisfied". 
de se respeitar o requisito da informação, entendido como a necessidade de fornecer ao indivíduo toda a informação e dados relativos à investigação ou ensaio clínico para a qual se pretende a sua participação;39 em segundo lugar, essa informação terá de ser transmitida de forma totalmente compreensível e clara, de maneira a que o interessado possa conhecer fácil e claramente todos os fatos, fatores, benefícios, riscos, natureza, objetivo, etc.; por fim, que a decisão que tome o indivíduo não seja influenciada ou coagida, ou que se preste sobre os efeitos de qualquer tipo de pressão, ou seja, essa decisão se exige livre e voluntária. ${ }^{40}$

\title{
4.3 Os princípios bioéticos de Beauchamp e Childress
}

No entanto, os princípios definidos pelo Relatório de Belmont só alcançariam uma maior difusão e reconhecimento a partir da publicação, em 1979, da obra Principles of Biomedical Ethics, da autoria de Tom L. Beauchamp (que tinha sido membro ativo da Comissão que compôs e redigiu o Relatório de Belmont) e de James F. Childress (teólogo e filósofo). Nessa obra, os autores pretenderam abordar uma recente e inovadora teoria que permitiria justificar a manifestação de uma nova ética médica, especificamente centrada na Biomedicina, ao considerar que a tradicional, a ética médica, era insuficiente e incapaz de dar uma resposta convincente, eficaz e inequívoca aos problemas surgidos e provocados pelas novas metodologias, técnicas e conhecimentos científicos. Para tal, formularam um modelo teórico baseado em princípios imperativos (prima facie), ainda que não absolutos. Na opinião de Velasco Gogenola (2009, p. 152-153),

\begin{abstract}
Estos son concebidos como el referente primigenio que indicará la moralidad de las decisiones que se tomen en biomedicina, pero de una manera determinada. En un primer momento, son considerados como principios prima facie, es decir, obligatorios sí, pero no absolutamente, pues es necesario tener en cuenta todas las circunstancias que concurren en la acción para establecer un juicio correcto; de esta manera, en caso de conflicto entre los principios, habría que dilucidar cuál es el que prevalece sobre los otros y determina la conducta a seguir, pues en el principialismo diseñado por Beauchamp y Childress, tanto el respeto a la autonomía como la beneficencia, la no-maleficencia y la justicia tienen el mismo rango y categoria. ${ }^{41}$
\end{abstract}

A partir da obra de Beauchamp e Childress podem ser obtidos quatro grandes princípios de ética médica: o princípio da autonomia (que o Relatório de Belmont definia como princípio de respeito pela pessoa), segundo o qual se confere aos pacientes uma maior participação no processo decisório ao considerá-lo como um sujeito "autônomo" e totalmente capacitado para decidir sobre a sua pessoa, sobre a sua vida e sobre o seu futuro. Com o objetivo de garantir essa participação no processo decisório, os autores reclamam e exigem a imperatividade da obtenção do consentimento informado de todas aquelas pessoas que se submeterão à investigação ou ensaio clínico; o princípio de beneficiência, igualmente previsto no Relatório de Belmont, através do qual se impõe a obrigação de obter as maiores vantagens para os pacientes, ou seja, que toda a atuação médica esteja dirigida e orientada a proporcionar aos sujeitos todos os possíveis benefícios alcançados pela intervenção e investigação biomédica; o princípio de nãomaleficência, um princípio formulado pelos autores por primeira vez, que impõe a obrigação geral de não causar dano a nenhuma pessoa e que, a partir de uma perspectiva ética-médica, proíbe qualquer tipo de conduta que ofenda ou vá contra os interesses dos pacientes, provocando-Ihes determinado tipo de dano

39 Segundo o mencionado relatório: "Comprehension. The manner and context in which information is conveyed is as important as the information itself. For example, presenting information in a disorganized and rapid fashion, allowing too little time for consideration or curtailing opportunities for questioning, all may adversely affect a subject's ability to make an informed choice. Because the subject's ability to understand is a function of intelligence, rationality, maturity and language, it is necessary to adapt the presentation of the information to the subject's capacities. Investigators are responsible for ascertaining that the subject has comprehended the information. While there is always an obligation to ascertain that the information about risk to subjects is complete and adequately comprehended, when the risks are more serious, that obligation increases. On occasion, it may be suitable to give some oral or written tests of comprehension".

$40 \quad$ Nas palavras do documento anteriormente citado: "Voluntariness. An agreement to participate in research constitutes a valid consent only if voluntarily given. This element of informed consent requires conditions free of coercion and undue influence".

41 Cfr. Velasco Gogenola (2009, p. 152-153). Nesse mesmo sentido parece ir Atienza (1998, p. 76), quando considera que "se trata de principios prima facie, esto es, que obligan siempre y cuando no entren en conflicto entre sí en caso de conflicto, los principios se jerarquizan a la vista de la situación concreta; o, dicho de otra forma, no hay reglas previas que den prioridad a un principio sobre otro, y de ahí la necesidad de llegar a un consenso entre todos los implicados, lo que constituye el objetivo fundamental de los comités institucionales de ética". 
com essa ação; ${ }^{42}$ por último, o princípio de justiça, que adverte para a necessidade (obrigatória) de que todos os pacientes recebam um tratamento justo e de acordo com o caso concreto ou, por outras palavras, um tratamento equitativo de todos os pacientes (GARCÍA, 2013, p. 69). ${ }^{43}$

\section{Conclusão}

Apesar dos nefastos acontecimentos que marcaram o século passado, com todas as atrocidades cometidas e as gravíssimas violações dos direitos fundamentais, o estudo e análise da Bioética acabou por sofrer um notável incremento. Seja por iniciativa de entidades governamentais (como foi o caso do Relatório de Belmont), seja por iniciativa de autores interessados na matéria (como foi o caso de Beauchamp e Childress), é inegável o avanço registrado na inclusão dos princípios bioéticos no âmbito das investigações biomédicas como mecanismo de defesa dos direitos e interesses dos pacientes.

Assim, concluindo esse resumido estudo sobre os principais episódios que estiveram na gênese dos princípios bioéticos formulados por Beauchamp e Childress (incluídos na segunda parte da sua obra, e que se dedica aos Princípios Morais), ${ }^{44}$ entendemos que, hoje em dia, esse catálogo de princípios deve ser considerado como o principal guia orientador e de forçoso respeito no que se refere à moralidade e ética no curso de investigações biomédicas; e que, em última instância, possibilita uma maior defesa e proteção dos sujeitos que se submetem a esses procedimentos e, consequentemente, mais avanços técnico-científicos (e até terapêuticos).

Portanto, não é exagerado considerar que o atual estado da disciplina da Bioética se deve, em enorme medida, à obra Principles of Biomedical Ethics e a tudo o que se criou a partir desse momento, seja através de estudos em defesa das ideias ali abordadas e expostas, seja através de teses contrárias e opostas. Finalmente, como consequência lógica dessa dicotomia, não se pode obviar a criação de distintas escolas de pensamento ético-moral. ${ }^{45}$

\section{Referências}

ÁLVAREZ-DÍAZ, Jorge Alberto. Los inicios de la bioética en Estados Unidos. In: HERREROS RUIZVALDEPEÑAS, Benjamin; BANDRÉS MOYA, Fernando (Coord.). Historia ilustrada de la Bioética. [S.I.]: ADEMAS Comunicación Gráfica, 2015. p. 147-158.

ATIENZA, Manuel. Juridificar la Bioética. Isonomía: Revista de teoría y filosofía del derecho, Cidade do México, Instituto Tecnológico Autónomo de México, n. 8, p. 75-99, 1998.

BRANDT, Allan M. Racism and research: The case of the Tuskegee Syphilis Study. The Hastings Center Report, Nova lorque, v. 8, n. 6, p. 21-29, 1978.

42 Por nossa parte, entendemos, de novo, que esse princípio mais não é que uma extensão ou expressão do princípio tradicional primum non nocere.

43 No mesmo sentido, mas em obra distinta, o autor indica que "Estos autores proponian los 'cuatro principios': autonomía, beneficencia, no maleficencia, y justicia [...]. La autonomía hace referencia al deber de respetar la capacidad de toma de decisiones del individuo, permitiendo que tomen decisiones razonadas e informadas. La beneficencia se refiere al deber que tienen el personal médico y las estructuras sanitarias de actuar siempre en beneficio del paciente. Por el contrario, la no maleficencia apunta al deber de evitar causar daño al paciente: el daño que pueda producir un tratamiento tiene que ser proporcionado y menor que el beneficio que produce. Y finalmente, la justicia indica que se tienen que distribuir los riesgos, daños, beneficios y costes en un modo ecuo: no se puede tratar a pacientes que estén en situaciones semejantes de modos distintos". (GARCÍA, 2010).

44 'La segunda parte, 'Principios morales', que sería el núcleo del libro, consta de cinco capítulos en los cuales los autores presentan los llamados cuatro principios de la bioética: el respeto a la autonomía (una acción autónoma es actuar intencionadamente, con conocimiento, sin influencias externas), la no maleficencia (no hacer daño intencionadamente), la beneficencia (obligación moral de actuar en beneficio de los otros) y la justicia (las personas que tienen derechos iguales tienen que ser tratadas con igualdad, y las personas que no tienen derechos iguales tienen que ser tratadas de manera diferenciada -justicia formal-; distribución equitativa de los derechos y de responsabilidades o cargas en la sociedad -justicia distributiva-)". (MIR TUBAU; BUSQUETS ALIBÉS, 2011, p. 3).

45 Como pode ser, a título de exemplo, a escola do Principialismo, do Casuísmo, do Utilitarismo ou das Éticas da virtude. Para uma breve aproximação dessas correntes filosóficas que se desenvolvem especialmente a partir da obra de Beauchamp e Childress, Cfr. Herreros (2015, p. 169 e ss). 
CASTRO MORENO, Julio Alejandro. Eugenesia, genética y bioética. Conexiones históricas y vínculos actuales. Revista de Bioética y Derecho, Barcelona, n. 30, p. 66-76, enero 2014.

CUERDA-GALINDO, E.; SIERRA-VALENTI, X.; GONZÁLEZ-LÓPEZ, E.; LÓPEZ-MUÑOZ, F. La sífilis y la experimentación en humanos: perspectiva histórica y reflexiones éticas. De la Segunda Guerra Mundial a la actualidad. Actas Dermo-Sifiliográficas, Barcelona, v. 105, n. 8, p. 809-886, 2014.

DE ABAJO IGLESIAS, Francisco José. La Declaración de Helsinki IV: una revisión necesaria, pero ¿suficiente? Revista Española de Salud Pública, Madrid, v. 75, n. 5, p. 407-420, 2001.

DE ALMEIDA NEVES BARBAS, Stela Marcos. Direito do genoma humano. Coimbra: Almedina, 2007.

GARCÍA, Juan José. Bioética personalista y bioética principialista. Perspectivas. Cuadernos de bioética, Murcia, v. 24, n. 80, p. 67-76, 2013.

GARCÍA, Juan José. Bioética. In: FERNÁNDEZ LABASTIDA, Francisco; MERCADO, Juan Andrés (Ed.). Philosophica: Enciclopedia filosófica on line. Disponível em: < http://www.philosophica.info/voces/ bioetica/Bioetica.html>. Acesso em: 24 jul. 2018.

GUILLEM-TATAY, David. Historia de la Bioética. Observatorio de Bioética, Universidad Católica de Valencia, 30 oct. 2013. Disponível em: < https://www.observatoriobioetica.org/2013/10/historia-de-labioetica/554>. Acesso em: 24 jul. 2018.

MIR TUBAU, Joan; BUSQUETS ALIBÉS, Ester. Principios de Ética Biomédica, de Tom L. Beauchamp y James F. Childress. Bioètica \& debat, Barcelona, Tribuna abierta del Institut Borja de Bioètica, v. 17, n. 64, p. 3-7, 2011.

MORENO, Beatriz; VIVAS, Enrique; DE VEGA, Gerardo. Eugenesia, la moda del cambio de siglo. De Francis Galton a Joseph Mengele. In: HERREROS RUIZ-VALDEPEÑAS, Benjamin; BANDRÉS MOYA, Fernando (Coord.). Historia llustrada de la Bioética. [S.I.]: ADEMAS Comunicación Gráfica, 2015. p. 51-64.

PASCUAL, María Jesús; PACHO, Eloy. Del rigor alemán al desastre Nazi. La directiva Prusiana. In: HERREROS RUIZ-VALDEPEÑAS, Benjamin; BANDRÉS MOYA, Fernando (Coord.). Historia Ilustrada de la Bioética. [S.I.]: ADEMAS Comunicación Gráfica, 2015. p. 81-88.

ROMEO CASABONA, Carlos María. El Derecho penal ante el racismo y la eugenesia. Cuadernos del instituto Vasco de Criminología, San Sebastián, n. 11 extraordinario, p. 105-135, dic. 1997.

ROMEO CASABONA, Carlos María. La relación entre la bioética y el derecho. In: ROMEO CASABONA, Carlos María (Coord.). Derecho Biomédico y Bioética. Granada: Comares, 1998. p. 151-164.

SOUTULLO, Daniel. Eugenesia (Ético). In: ROMEO CASABONA, Carlos María (Dir.). Enciclopedia de Bioderecho y Bioética. Comunidade Autônoma Basca: Cátedra Interuniversitaria Fundación BBVADiputación Foral de Bizkaia de Derecho y Genoma Humano, Editorial Comares, Bilbao-Granada, 2011. t. I a-h.

SUÁREZ ESPINO, María Lidia. El derecho a la intimidad genética. Madrid: Marcial Pons, 2008.

THOMAS, Stephen B.; QUINN, Sandra Crouse. Light on the shadow of the syphilis study at tuskegee. Health Promotion Practice, Sage Publications, v. 1, n. 3, p. 234-237, 2000.

VELASCO GOGENOLA, Juan María de. Origen y desarrollo de una ciencia: la bioética. Revista internacional de los estudios vascos, Donostia:Sociedad de Estudios Vascos, Eusko Ikaskuntza,v. 54, n. 1, p. 147-187, 2009.

VILCHIS ROA, Derik Geovani. De Núremberg a Helsinki. Hans Joachim Sewering. In: HERREROS RUIZ-VALDEPEÑAS, Benjamín; BANDRÉS MOYA, Fernando (Coord.). Historia llustrada de la Bioética. [S.I.]: ADEMAS Comunicación Gráfica, 2015. p. 89-96. 